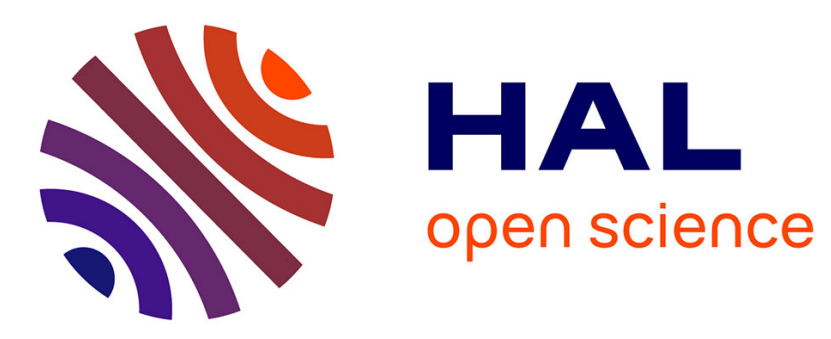

\title{
Reconstitution des paysages autour de Lucques, VIIIe-Xe siècles
}

\author{
Anne Mailloux
}

\section{To cite this version:}

Anne Mailloux. Reconstitution des paysages autour de Lucques, VIIIe-Xe siècles. Travaux de l'Ecole doctorale d'Histoire, 1998, Paris, France. pp.103-114. halshs-00663800

\section{HAL Id: halshs-00663800 \\ https://shs.hal.science/halshs-00663800}

Submitted on 19 Jan 2022

HAL is a multi-disciplinary open access archive for the deposit and dissemination of scientific research documents, whether they are published or not. The documents may come from teaching and research institutions in France or abroad, or from public or private research centers.
L'archive ouverte pluridisciplinaire HAL, est destinée au dépôt et à la diffusion de documents scientifiques de niveau recherche, publiés ou non, émanant des établissements d'enseignement et de recherche français ou étrangers, des laboratoires publics ou privés. 


\title{
RECONSTITUTION DES PAYSAGES AUTOUR DE LUCQUES, VIII ${ }^{\mathrm{e}} \mathrm{X}^{\mathrm{e}}$ SIÈCLES
}

\author{
Anne MAILLOUX*
}

Si l'on se tient à la définition donnée par Alain Roger dans son Court traité du paysage, le paysage est défini comme une représentation de l'espace, ainsi que sa perception par l'homme'. Le plaisir esthétique est ici une notion essentielle, ainsi que celle de création, ou de re-création artistique. Le paysage est donc une représentation de l'espace en fonction d'une codification esthétique dont le but essentiel est de produire une oeuvre artistique.

Bien souvent, l'historien du paysage situe ses préoccupations bien en-deçà de cette approche esthétique de l'espace. Mon propos consiste essentiellement en une compréhension des structures de l'espace agraire, plus que d'un paysage agraire parfois difficile à définir dans ses composantes. Parler de paysage est donc légèrement abusif, sauf si l'on se place du point de vue de la perception généralement mise en œuvre lors des descriptions de biens dans les actes notariés. Toutefois, il nous sera consenti de parler de paysage dans la mesure où l'espace considéré n'est pas totalement dégagé des contraintes et des structures imposées par l'homme, de l'aménagement et de la trace humaines.

Dans ma thèse, le but était de comprendre les structures d'un territoire, le diocèse de Lucques, et sa mise en place, grâce à l'examen du mécanisme institutionnel de gestion du territoire, et de comprendre en parallèle les structures générales de l'implantation humaine (habitat, réseau viaire...), d'analyser les pratiques spatiales d'une institution particulière, l'évêché de Lucques, ses stratégies patrimoniales et ses choix de mise en valeur agricole, d'éclairer cette analyse des caractéristiques du parcellaire foncier de la Lucchesia lombarde et carolingienne. L'ensemble de ces interrogations est nourri par l'existence d'un fonds archivistique riche et continu chronologiquement, suffisamment fourni pour suivre précisément l'évolution des modes de représentation de l'espace, les rythmes de constitution et de mise en valeur du patrimoine ecclé-

\footnotetext{
* A soutenu une thèse en histoire médiévale : Lucques et son territoire, des Lombards aux Ottoniens (685-1000). Structures du patrimoine épiscopal.

1. Cf. A. Roger, Court traité du paysage, Paris, 1997.
} 
siastique, et enfin les évolutions des structures de l'espace (habitat, trames viaires, formes et évolutions du parcellaire, ...).

En effet, le fonds de l'Archivio Arcivescovile di Lucca comprend pour la période du $\mathrm{VIII}^{\mathrm{e}}$ au $\mathrm{X}^{\mathrm{e}}$ siècle (685-1000) 1800 parchemins, supports d'actes notariaux fixant les transactions foncières de l'évêché $e^{2}$. Une des principales caractéristiques des mutations foncières et des contrats agraires ainsi conservés réside dans le soin minutieux porté à la description de l'espace agraire et des cultures. D'autres sources permettent d'envisager une tentative de reconstitution, aussi schématique soit-elle, des paysages lucquois du haut Moyen Âge : les traces persistantes de la centuriation romaine dans la plaine de Lucques, ainsi que les cartes du cadastre établi à Lucques entre 1820 et 1840 .

Je m'attacherai ici à développer deux aspects, qui correspondent à deux échelles d'appréhension de l'espace. Elles sont suggérées par le fonctionnement même des actes et leur typologie : le système de description des biens en est le révélateur le plus sûr ${ }^{3}$. Deux systèmes concurrents de description de l'espace coexistent dans les actes lucquois : une description par énumération des éléments de la propriété ou de l'exploitation, et un second système par confronts, qui s'attache à une description topographique et patrimoniale des parcelles. Les deux systèmes de description conditionnent ces deux échelles d'appréhension de l'espace.

Une image globale des paysages émerge, et l'on peut obtenir, en particulier, une idée de la répartition des cultures par masse et du rapport existant entre les différents types de mise en valeur agraire. D'autre part, grâce à la description en détail de certaines parcelles, notamment en

2. Pour l'ensemble de la période, les actes conservés se répartissent selon la typologie suivante : la très grande majorité des transactions est composée de 4 types (sur 12 déterminés), livello $(54,6 \%)$, ventes $(6,5 \%)$, donations $(14,2 \%)$, échanges de biens $(11,2 \%)$. Une part non négligeable dans le fonds épiscopal est occupée par les actes conservant la mémoire des concessions d'églises, privées ou plébanes (plus de $8 \%$ des actes conservés). Le reste des sources est composé de documents divers (notices de jugement, 2,3\%, inventaires de biens, $0,4 \%$, opérations de crédit, $0,2 \% \ldots$.

3. Cf. A. MaillouX, «Perception de l'espace chez les notaires de Lucques (VIII ${ }^{\mathrm{e}}-\mathrm{IX}^{\mathrm{e}}$ siècle) », MEFRM, 109-1 (1997), p. 21-57. Le système de description des biens choisi par le notaire répond à la fonction de l'acte établi. Trois cas sont envisageables : dans les transactions où la valeur marchande du bien est le fondement même de l'opération (vente ou échanges de biens), les éléments de la description (dans la très grande majorité des cas par confronts) sont extrêmement précis. La fixation de la valeur dépend de ces facteurs objectifs que constituent la localisation du bien, sa surface et la qualité de la terre ou des bâtiments construits (dans les ventes, les descriptions par confronts atteignent $43 \%$, par énumération $56 \%$, dans les échanges de biens, ces chiffres atteignent respectivement 80 et $18 \%$ ). Les partages de biens soulignent régulièrement les aspects patrimoniaux, délimitation et localisation des parcelles, rappel de la situation juridique antérieure... Enfin, les contrats agraires mettent plutôt en évidence la localisation du bien, le type de mise en valeur agricole et les revenus que l'on espère en tirer. Dans les deux premiers cas, une description par confronts est plus adaptée, alors que dans les contrats agraires, l'énumération de biens permet de mieux inventorier les éléments consitutifs de l'exploitation concédée (75,5\% de descriptions par énumération, $6 \%$ par confronts). 
milieu de plaine, il est possible d'obtenir une image précise du parcellaire foncier et de ses évolutions.

Le territoire du diocèse de Lucques est composé d'espaces assez variés dans leur morphologie et leur végétation. On y observe successivement, autour de la cité, une plaine bordée de collines qui marquent au sud la frontière avec le diocèse de Pise. Au nord et à l'ouest, les reliefs s'accentuent brutalement, pour atteindre plus de $1000 \mathrm{~m}$ d'altitude dans la Garfagnane et la terminaison des Alpes Apuanes. Combiné à ces caractéristiques, le régime des eaux constitue une contrainte majeure dans cette région de la Toscane septentrionale. Le Serchio, après un parcours encaissé dans les montagnes, s'étale dans la plaine, ainsi que les nombreux petits torrents qui inondent l'ensemble de la plaine de Lucques. Des lacs et des zones marécageuses, à l'exutoire de ces cours d'eau, constituent une contrainte supplémentaire.

Je voudrais ici m'attacher à l'étude d'un terroir particulier, celui de Lunata, dans la plaine, où la qualité des renseignements disponibles permet de redessiner assez précisément les grandes lignes du paysage et du parcellaire, ainsi que ses évolutions, patrimoniales et parcellaires.

\section{Esquisse du paysage}

Dans les descriptions par énumération, le notaire et les parties procèdent en détaillant les biens par genre, bâtiments et cultures. Ils suivent un ordre presque immuable: dans un premier temps sont mentionnées les caractéristiques générales de l'exploitation ou de la propriété (maison et biens), puis les éléments composant cet ensemble, en partant des modules les plus proches de l'habitat (bâtiments d'exploitation), puis en précisant tous les éléments agraires composant l'exploitation. La formule " maison, bâtiments (parfois précisés), cour, jardin, terres, vignes, bois, vergers, prés et pacages », est la plus fréquente. Quelques termes plus généraux permettent d'englober tous les biens dépendants (biens mobiliers, immobiliers...).

Le formulaire de description par énumération est de loin le plus couramment employé dans les actes lucquois. Malgré la standardisation du formulaire, il est possible d'effectuer un comptage des occurrences des différents éléments du paysage. J'ai remarqué que les descriptions ne sont jamais totalement figées. À l'intérieur d'un cadre très formalisé, la description s'efforce de rendre compte réellement des éléments constitutifs des propriétés et exploitations agricoles en jeu: jamais les châtaigniers n'apparaissent en plaine, tout comme les vignes ou les oliviers en haute montagne. Par ce procédé (comptage de la proportion relative des éléments du paysage, cultures, habitat, réseaux...), on obtient une esquisse du paysage. 


\section{Habitat}

Dans les énumérations, la graduation du plus précis au plus général, du plus proche au plus éloigné spatialement, est en soi intéressante: l'espace est organisé autour de l'unité d'habitation et d'exploitation. Cette perception de l'espace semble indicative d'une installation diffuse dans l'espace, sans groupement très fort de l'habitat. L'exploitation agricole (casa et res) apparaît dans les contrats agraires comme l'unité de base de la mise en exploitation de l'espace.

L'association systématique des terres et de l'habitat est l'élément le plus caractéristique : dans les contrats agraires, les concessions sans maison sont rares, et inversement les maisons, avec toute la variation de vocabulaire spécifique à l'habitat, sont presque toujours citées en première place de l'énumération. L'habitat est un élément fondamental de l'exploitation agricole.

La disposition relative de l'habitat par rapport aux terres est difficile à déterminer à partir de ces seules descriptions, sauf dans le cas où des expressions spécifiques désignent des terres accolées directement à l'habitat: ainsi, les expressions clausura, et totum insimul admembrata, employées pour décrire le premier cercle autour de la maison.

On pourrait ébaucher l'image d'un habitat dispersé, accru de bâtiments annexes, d'une cour, éventuellement d'un jardin, le tout parfois entouré de haies, et muni d'un accès ${ }^{4}$. Les termes de casale, casalino, renvoient d'ailleurs probablement à cette réalité et sont toujours employés pour décrire l'habitat rural dispersé. Dans certains cas, le bâtiment est qualifié du terme de capanna. L'habitat de moindre taille est donc soigneusement décrit, et la richesse du vocabulaire spécifique de l'habitat est révélatrice de l'importance qu'il revêt dans l'esprit des contractants.

Ce premier constat semble toutefois en partie erroné : les actes trahissent, en même temps que l'image d'un habitat relativement lâche, l'émergence d'une identité territoriale très nette, dès le VIII ${ }^{\mathrm{e}}$ siècle, centrée le plus souvent autour de l'église plébane. C'est notamment le cas dans les parties montagneuses du diocèse, mais aussi, de manière beaucoup plus surprenante, en zone de plaine, où l'ensemble des structures décelables reflètent plutôt l'image d'un habitat diffus. Si l'on ne peut parler de village groupé, il existe toutefois un noyau d'habitat suffisamment dense pour constituer un point focal de l'identité territoriale et de l'implantation humaine. Nous verrons que les patrimoines sont plutôt éclatés dans la plaine, notamment au VIII ${ }^{\mathrm{e}}$ siècle, que les cultures sont très imbriquées les unes aux autres, sans formation de zones de monoculture. On ne peut pour autant en déduire qu'il en va de même pour l'habitat,

4. Dès le début du VIII' siècle, la loi lombarde attache une importance particulière à la protection de cette unité de base, où toute intrusion est sévèrement punie. Cf. C. AzzARA et S. GASPARRI, Le leggi dei Longobardi. Storia, memoria e diritto di un popolo germanico, Milan, 1992. 
que l'on peut par conséquent qualifier de semi-groupé. Les descriptions par énumération, par conséquent, s'attachent plus à rendre compte d'une réalité organique, et non une entité spatiale groupée et cohérente.

\section{Végétation et cultures}

Les proportions de chaque type de culture soulignent la diversité qui préside dans l'espace lucquois (champs emblavés, prés, pacages, vignes, oliviers, arbres, châtaigniers...).

À l'évidence, la base de toute exploitation est constituée du duo terres - vignes ${ }^{5}$. La place de la vigne dans le paysage lucquois, et dans son économie, est primordiale : si les terres et les vignes ne tiennent pas la même proportion dans les paysages, la vigne prend une valeur économique telle que sa présence est systématiquement signalée dans l'inventaire des biens effectué lors de la transaction. Il est plus difficile en revanche de parler de trilogie méditerranéenne, impliquant un relatif équilibre entre ses trois éléments (blé, vigne, olivier) : le développement de la culture intensive de l'olivier n'advient qu'au $\mathrm{XI}^{\mathrm{e}}$ siècle ${ }^{6}$. La mention d'oliviers est auparavant limitée dans les actes lucquois, même si les redevances en part de récolte d'olives existent. Pour notre période, c'est la vigne qui est la culture spéculative par excellence ${ }^{7}$. Les oliviers, chênes, châtaigniers sont cités plus ou moins sporadiquement en fonction de la situation géographique du bien concédé.

Les contrats agraires permettent enfin de déceler des pratiques nouvelles d'aménagement de parcelles (vignes, prés à fourrage dans les zones marécageuses), dès le $\mathrm{IX}^{\mathrm{e}}$ siècle. $\mathrm{Au} \mathrm{X}^{\mathrm{e}}$ siècle, des mentions de plus en plus fréquentes de sterpeto $^{8}$ traduisent un élargissement de l'espace en culture, souvent parallèle à la mise en valeur des castelli nouvellement organisés.

\section{La mosaïque parcellaire et patrimoniale : le cas de Lunata}

Le système de description par confronts (capita et latera) est employé lorsque les transactions portent sur des parcelles et biens délimités. Les limites sont alors soigneusement désignées, voire matérialisées (quelques opérations d'arpentage sont rappelées, le plus souvent lors de

5. Il reste toujours difficile d'affirmer que le terme très général de terre signifie terre labourée ou emblavée. C'est très souvent le cas, sans pour autant que l'on puisse exclure le sens de terrain, pièce de terre. Cette remarque vaut pour les descriptions par énumération aussi bien que pour celles par confronts.

6. Cf. C. Wickham, Comunità e clientèle nella Toscana del XII secolo. Le origini del comune rurale nella Piana di Lucca, Rome, 1995.

7. On rejoint ici les considérations de L. Feller, Les Abruzzes médiévales. Territoire, économie et société en Italie centrale du IXe au XII 'e siècle, Rome (B.E.F.A.R. 300), 1998.

8. Sterpeto : essart. Cf. stirpetum, J. F. NIERMEYER, Mediae Latinitatis Lexicon Minus, Leyde, 1976, p. 993. 
partages de biens, ou, au $\mathrm{X}^{\mathrm{e}}$ siècle, lors d'échanges de grande ampleur préalables à la constitution de castra). Les cultures et parfois la surface sont le plus souvent mentionnées, les parcelles adjacentes sont elles-aussi décrites (propriétaire et culture) ${ }^{9}$. Il est ainsi possible de reconstituer un « schéma de l'occupation du sol » dans certaines zones où la documentation est plus fournie. On peut même suivre les variations patrimoniales, parfois noter l'évolution des parcelles. Les descriptions par confronts sont adaptées à des formes quadrilatérales (carrés, rectangles, trapèzes). Dans leur très grande majorité, ces parcelles quadrilatérales sont localisées dans la plaine. Selon les périodes, le formulaire varie légèrement: on observe ainsi à partir du $\mathrm{X}^{\mathrm{e}}$ siècle une nette tendance à décrire par ce système des parcelles non plus quadrilatérales, mais de forme irrégulière ( 5 ou 6 côtés), le plus souvent qualifiées de sterpeto.

Ce type de description est éminemment adapté à la structure spatiale de la plaine, caractérisée par un maillage très dense. C'est dans cet espace que l'on peut se poser au maximum le problème de la continuité des structures de l'espace et de la perception de l'espace, par rapport à l'époque romaine.

En effet, une opération de centuriation de Lucques, d'après les éléments rapportés par Pline, semble remonter à $180 \mathrm{av}$. J.-C., et une seconde déduction, effectuée sous le triumvirat d'Octave, Antoine et Lépide, semble avoir repris le tracé de la première. Les repérages de la centuriation ne permettent pas de remarquer de solution de continuité entre ces deux opérations distantes de plus d'un siècle. 70 à 80 centuries ont été repérées grâce à des clichés de la RAF, ainsi que certains kardines et decumani, dont les plus visibles (y compris sur les cartes topographiques actuelles au 1/25 000), comme le Fosso Formicola, se situent au sud de la cité lucquoise. Pour autant, les marques pérennes de la centuriation semblent perturbées par les divagations des cours d'eau et leur variation séculaire. C'est notamment le cas dans la zone de Lunata, qui devait se situer sur une boucle du Serchio ${ }^{10}$.

Le cas de Lunata a attiré précocément mon attention, dans la mesure où nous disposons pour ce petit territoire, peu distant de Lucques, d'un nombre fourni de sources, variées typologiquement. Plusieurs dossiers patrimoniaux ${ }^{11}$ regroupés aux archives épiscopales par versement

9. Cf. A. Mailloux, Perception, art. cit.

10. Sur les problèmes de la centuriation lucquoise, cf. F. CASTAGNOLI, « La centuriazione di Lucca », Studi Etruschi, XX, Florence, 1848-48, p. 285-290, et A. MAIllouX, « Pour une étude des paysages dans le territoire de Lucques au haut Moyen Âge (VIII siècle) ", dans De la terre au ciel. I. Paysages et cadastres antiques. (XII ${ }^{\mathrm{e}}$ stage international, Besançon, 29-31 mars 1993), M. Clavel-LévÊque, I. JoufFroy, A. Vignot éd., Paris, 1994, p. 207-222. Je renvoie à la cartographie comprise dans ces deux articles. Les problèmes liés aux variations du Serchio sont évoqués dans l'article de Castagnoli.

11. Le dossier le plus connu est celui de Crespin, négociant lucquois. Cf. B. ANDREolli, Uomini nel Medioevo. Studi sulla società lucchese dei secoli VIII-XI, Bologne, 1983, et notamment les p. 33 à 38 , consacrées à ce personnage. Ce personnage, après avoir cons- 
lors de l'acquisition des parcelles par cette institution aux $\mathrm{VIII}^{\mathrm{e}}$ et $\mathrm{IX}^{\mathrm{e}}$ siècles mettent en évidence les nombreuses opérations patrimoniales de l'évêché dans ce territoire. L'armature plébane est stable et permet de contrôler plusieurs églises privées, réparties sur un territoire restreint. Le recteur de l'église plébane San Frediano occupe une place importante dans la hiérarchie curiale, dès le VIII $^{\mathrm{e}}$ siècle $^{12}$. Une trentaine de documents relatifs à la gestion du patrimoine de l'église plébane San Frediano de Lunata soulignent la place occupée par cette plebs de plaine dans les préoccupations de l'évêché.

La confrontation des renseignements fournis par les analyses de la matrice centuriale et des documents relatifs à Lunata fournit quelques indications précieuses. Dans cette zone, la matrice de la centuriation a subi quelques perturbations pendant l'Antiquité, puisque le cours principal du Serchio devait passer à cet endroit. Pour autant, l'ensemble des parcelles décrites par confronts reflètent l'image d'un parcellaire régulier, caractérisé par des modules quadrilatéraux, où les cours d'eau, chemins et haies servent souvent de limites. La constitution du parcellaire, postérieure au changement de cours du Serchio, semble donc suivre, sinon un tracé identique, du moins des modalités similaires à la constitution du parcellaire en milieu centurié. On peut envisager, avec quelques précautions toutefois, que les structures de la centuriation ont été prolongées pour coloniser les espaces libérés par la variation des cours d'eau. Quoi qu'il en soit, le système de description des parcelles suit ici un cours très imprégné des pratiques gromatiques antiques ${ }^{13}$. Le bilan peut donc être nuancé : il n'y a pas de continuité totale, même si la construction du paysage agraire au début du Moyen Age suit des modalités très fortement imprégnées de tradition antique. Les innovations liées aux transformations du milieu, que l'on peut dater de la fin du $\mathrm{VI}^{\mathrm{e}}$ siècle $^{14}$, s'appuient sur l'ancienne trame du paysage.

Il est possible de reconstituer un schéma de l'aménagement des parcelles et de l'agencement des propriétés dans la zone de Lunata. Je propose ici une reconstitution schématique du parcellaire, d'après quelques actes concernant la zone de Lunata ${ }^{15}$.

titué un patrimoine de quelque importance, fonde une église privée (Saint-Martin de Lunata) qu'il dote et transmet à son fils Teuselmo prêtre. Le domaine et l'église tombent dans l'escarcelle de l'Église lucquoise à la fin du VIII' siècle.

12. C'est notamment le cas de Austrifonso diacre, recteur de San Frediano di Lunata, qui est très actif dans la gestion du patrimoine qui lui a été confié, ainsi que son successeur Jean prêtre.

13. Cf. A. Mailloux, "Perception », art. cit., notamment p. 39-46.

14. Cf. A. Mailloux, «Pour une étude des paysages», art. cit., à propos du miracle de san Frediano et de la dévotion particulière des habitants de Lunata pour cet évêque du milieu du VI ${ }^{\mathrm{c}}$ siècle. Cf. aussi P. SQuATRITI, «Water, Nature and Culture in Early Medieval Lucca », Early Medieval Europe, vol. 4, 1 (1995), p. 21-40.

15. Les actes cités peuvent être consultés dans l'édition ancienne, et parfois lacunaire, mais toujours précieuse, de D. BARSOCCHINI, Memorie e documenti per servire alla storia 
AAL 41 : mars 752, vente de biens. Ce cas de figure représente le type de plus simple de reconstitution possible.

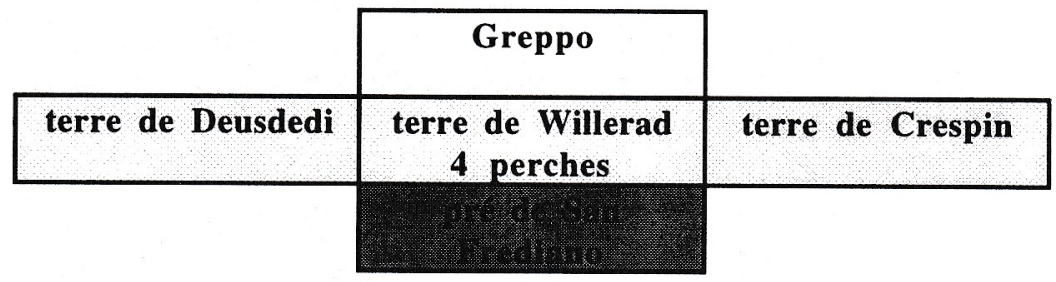

AAL 90 : 29 juin 764, donation de deux ensembles de parcelles, lieudit « Ussiano ».

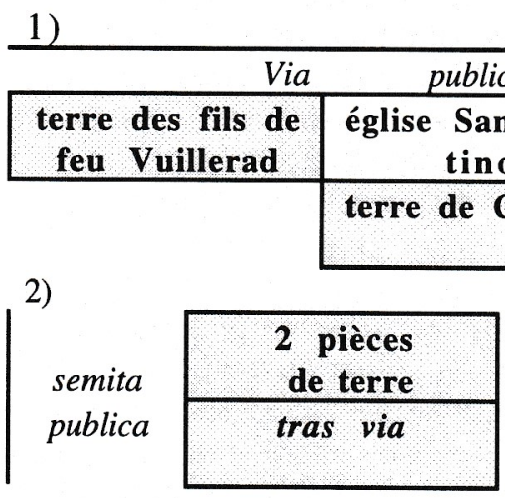

Ici, les deux groupes de parcelles pourraient être conjoints, si l'on accepte que la semita publica est identique à l'une des deux via publica longeant l'église Saint-Martin. Deux agencements sont possibles :

1)

\begin{tabular}{|c|c|c|}
\hline $\begin{array}{l}2 \text { pièces } \\
\text { de terre }\end{array}$ & tras via & \\
\hline \multicolumn{2}{|r|}{ publica } & \multirow{3}{*}{$\begin{array}{c}\text { / semita p } \\
\text { via } \\
\text { publica }\end{array}$} \\
\hline $\begin{array}{l}\text { terre des fils de } \\
\text { feu Vuillerad }\end{array}$ & église $\underset{\text { San }}{\text { San }}$ Mar- & \\
\hline & terre de Crespin & \\
\hline
\end{tabular}

di Lucca, V, 2 et 3, Atti della Reale Accademia lucchese, Lucques, 1837-1841. Les actes antérieurs à 774 sont aussi disponibles dans l'édition de L. SchiaPARELLI, Codice Diplomatico Longobardo, Rome, 1929-1933 (Fonti per la Storia d'Italia, 62-63). 
ou 2)

\begin{tabular}{|c|c|}
\hline Via & publica \\
\hline $\begin{array}{l}\text { terre des fils de } \\
\text { feu Vuillerad }\end{array}$ & $\begin{array}{c}\text { église San } \\
\text { Martino }\end{array}$ \\
\hline & $\begin{array}{l}\text { terre de } \\
\text { Crespin }\end{array}$ \\
\hline
\end{tabular}

via
publica
/semita
publica

\begin{tabular}{|c|}
\hline 2 pièces \\
de terre \\
\hline tras via \\
\hline
\end{tabular}

AAL 107 : novembre 767, donation, lieu-dit Civiliano. L'imbrication des cultures apparaît, ainsi que celle des propriétés.

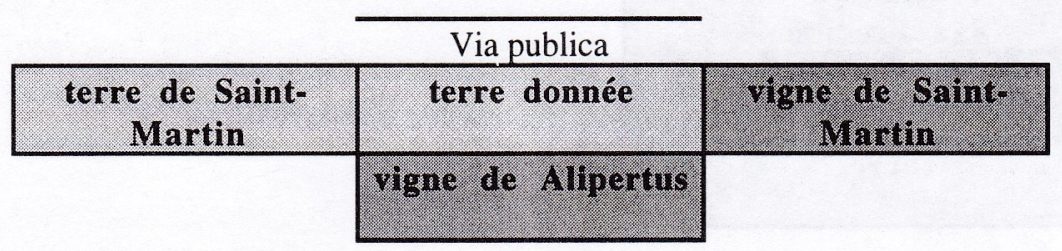

AAL 237 : $1^{\text {er }}$ juin 789, échange de parcelles en vue d'un remembrement. Par l'échange d'une parcelle, Argimo obtient un ensemble totalement cohérent, en contrepartie d'une perte de surface possédée. L'imbrication des propriétés, déjà notée, est ainsi corrigée par des réajustements portant sur de petites surfaces. Dans le cas cité, le terme de terra n'est pas indicatif d'un type de culture.

\begin{tabular}{|c|c|c|c|}
\hline & & $\begin{array}{c}\text { terre de San } \\
\text { Frediano }\end{array}$ & \\
\hline & $\begin{array}{c}\text { terre des fils de } \\
\text { Guntipert }\end{array}$ & $\begin{array}{c}\text { terre de San } \\
\text { Frediano } \\
(6 \text { setiers) }\end{array}$ & $\begin{array}{l}\text { terre de } \\
\text { Argimo }\end{array}$ \\
\hline $\begin{array}{c}\text { terre des fils de } \\
\text { feu Radeulus }\end{array}$ & $\begin{array}{c}\text { terre de Argimo } \\
(7 \text { setiers })\end{array}$ & \multicolumn{2}{|c|}{$\begin{array}{l}\text { terre de } \\
\text { Argimo }\end{array}$} \\
\hline & \begin{tabular}{|c|} 
terre de \\
Alateulo \\
\end{tabular} & & \\
\hline
\end{tabular}

Après l'échange, on obtient le schéma suivant :

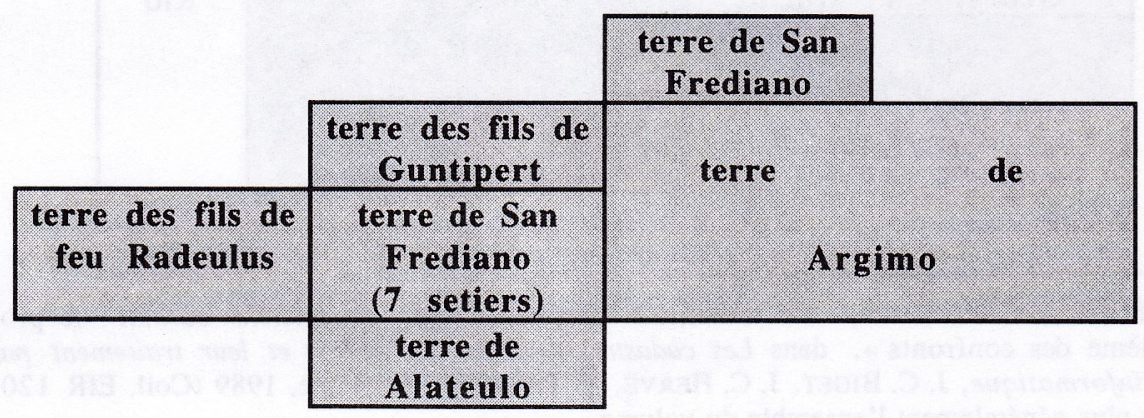


AAL 349 : décembre 806, vente, lieu-dit Ad puttio. Dans certains cas, je pense que la restitution de l'aménagement peut suivre le schéma proposé par É. Carpentier pour le cadastre d'Orvieto ${ }^{16}$ : lorsque deux confronts seulement sont cités, ils s'agit des côtés opposés de la parcelle. On peut alors juxtaposer les parcelles par bande.

\begin{tabular}{|c|}
\hline Rio Sana \\
\hline Pré de Cumpertus \\
\hline San Frediano \\
\hline pré \\
\hline terre ?
\end{tabular}

AAL 876 : 2 octobre 808, échange de biens.

\begin{tabular}{|c|c|c|c|}
\hline \multirow{3}{*}{$\begin{array}{c}\text { via } \\
\text { publica }\end{array}$} & \multicolumn{2}{|c|}{ "aqua } & publica" \\
\hline & $\begin{array}{l}\text { Yigne } \\
\text { de Tassulo }\end{array}$ & $\begin{array}{l}\text { vlghe } \\
\text { et salcuto }\end{array}$ & 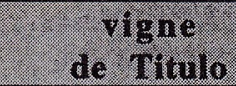 \\
\hline & & $\begin{array}{l}\text { terre des fils } \\
\text { de feu Pertualdo }\end{array}$ & \\
\hline
\end{tabular}

AAL 561 : 28 mai 838, vente, et AAL 679, 31 mars 848, donation. Ces deux documents portent sur les mêmes parcelles. On remarque que dans l'intervalle des dix ans écoulés, deux parcelles ont été regroupées, avec probablement un abandon de la vigne dans l'une des deux.

Premier état (838):

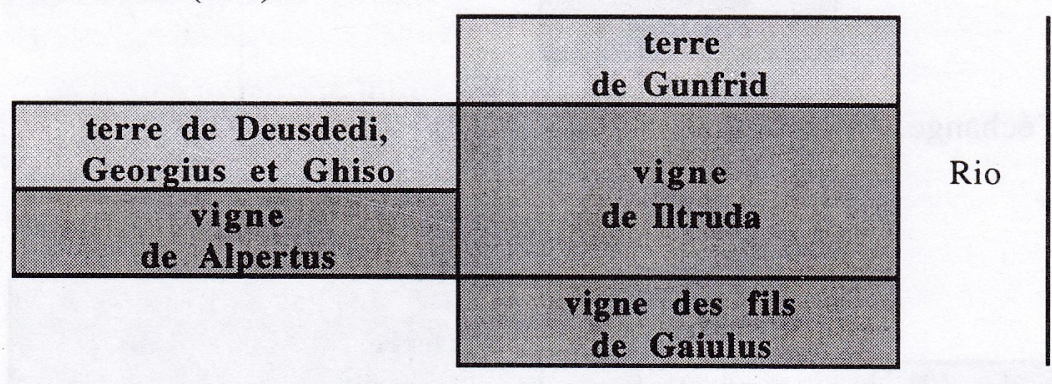

16. Cf. É. CARPENTIER, «Le traitement informatisé des plus anciens catasti: le problème des confronts », dans Les cadastres anciens des villes et leur traitement par l'informatique, J. C. BIGet, J. C. Hervé, Y. ThéBert éd., Rome, 1989 (Coll. EfR 120) et plus généralement l'ensemble du volume. 
Deuxième état (848) :

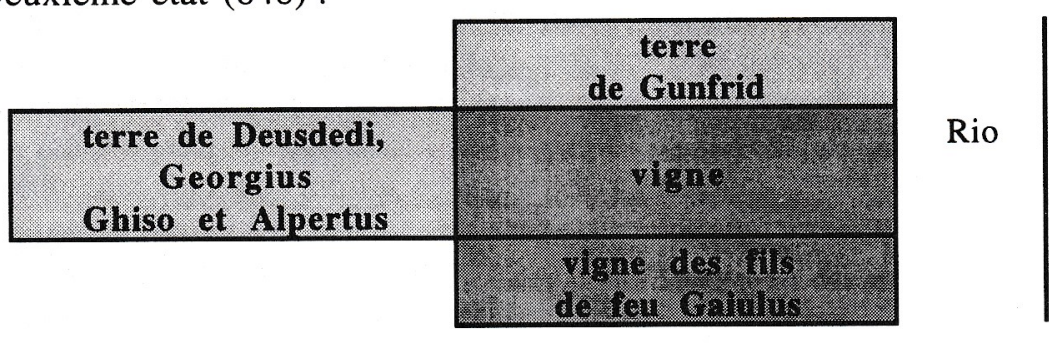

Plusieurs constatations s'imposent: l'éclatement de la propriété semble évident dans cette zone de plaine. De même, très rarement, les parcelles adjacentes font l'objet d'une même culture. On peut aussi souligner la probable petite taille des parcelles, ainsi que la régularité de leur forme, que je ne peux m'empêcher de mettre en relation avec les structures régulières de la centuriation (éventuellement prolongée dans les zones nouvellement conquises sur le Serchio). Les transactions nombreuses viennent corriger et améliorer ce schéma foncier. On observe ainsi une tendance au remembrement et à la réorganisation des propriétés. Ce processus de cohérence progressive touche aussi l'organisation des cultures. Le dernier élément notable est la densité de la trame formée par les cours d'eau et les chemins, qui contribue à la formation de ce micro-parcellaire que je qualifie volontiers de "mosaïque ».

L'analyse des parcellaires, de l'habitat et des cultures viennent affiner les questions que l'on se pose sur l'incastellamento lucquois et ses spécificités. En ce sens, le paysage est un moyen de comprendre un phénomène, de l'expliquer, avec toutefois une nuance: le paysage évolue, la dynamique qui l'anime permet de saisir d'autres mécanismes, sociaux et économiques aussi bien que politiques. On ne peut pour autant pas faire de déterminisme géographique, dans le sens que le paysage est tout autant un reflet du terrain que celui de l'activité humaine. Le paysage, et en particulier la structure que constitue le parcellaire, reflète la structure de la propriété et des patrimoines.

La source fondamentale dans mon étude reste le document d'archive notarié, même si la cartographie constitue un apport non négligeable à l'analyse. Il faut aussi souligner le caractère limité de ces reconstitutions : on obtient une vision biaisée des paysages, très influencée par le stock d'informations disponibles par zones: ainsi, à Lunata, l'expérience est possible grâce à l'abondance des sources (de type divers) et leur qualité. mais d'autres espaces aussi importants, comme Santa Maria a Monte, sont très difficile à reconstituer. Pourtant, il s'agit là d'un espace fondamental dans les stratégies épiscopales : les propriétés épiscopales ont tendance à se concentrer dans cette zone dès le $\mathrm{IX}^{\mathrm{e}}$ siècle. C'est sur ce territoire, aux confins des diocèses de Lucques et de Pise que l'évêque de Lucques constitue à la fin du IX ${ }^{\mathrm{e}}$ siècle un des premiers cas- 
telli de la Lucchesia, et un des centres majeurs de son administration. Le grand nombre d'actes relatifs à ce terroir est la preuve de l'importance de la localité dans le dispositif territorial et patrimonial de l'évêché dès cette date. On ne peut pas, à cause de la qualité des sources, déterminer si ces changements profonds dans l'équilibre territorial correspondent à une transformation en profondeur du paysage autour du château, aménagé de manière concentrée dès cette époque. Les renseignements sur le parcellaire sont ici très réduits. Le danger le plus évident serait celui de croire que la définition du paysage lucquois se réduit à celle des paysages de plaine. Le danger est d'autant plus fort qu'il existe une centuriation : tirer des conclusions (sur la continuité absolue et parfaite entre Antiquité et Moyen Âge) est une tentation à laquelle on ne peut succomber si l'on garde conscience que cette analyse n'est valide finalement que sur un espace assez restreint et déjà très organisé. 